\section{Benjamin Ibarra-Sevilla}

University of Minnesota

School of Architecture

89 Church St SE

Minneapolis, MN 55455, USA

ibarr002@umn.edu

Keywords: Mexican architecture, open chapel, sixteenth-century architecture, Teposcolula, symbolism, Euclidean geometry, harmonic proportions, geometric analysis, sacred geometry, spheres, trigonometry

\section{The Hidden Face of the Vault: Unveiling The Expression of the Avant-Garde Through the Use of the Sphere in Teposcolula's Open Chapel}

Presented at Nexus 2012: Relationships Between Architecture and Mathematics, Milan, 11-14 June 2012

Abstract. Benjamin Ibarra-Sevilla examines the role of the sphere in the Open Chapel built in San Pedro y San Pablo Teposcolula during sixteenth century. This unique Mexican building contains a complex ribbed vault designed on a hexagonal plan forming a semi-spherical dome. The investigation examines the use of the sphere in this structure in light of the design precepts practiced during the Renaissance in Europe. Furthermore, the study finds the connections of these precepts with a mathematical solution to the sphere based on convex shapes theories. By revealing the intricacies of the underlying geometry applied to design this building, this text aims to demonstrate the scholastic and architectural relevance of this refined Mexican piece of architecture.

\title{
Introduction
}

Circles and spheres have been the object of attention of architects and geometers for centuries, regardless of their origin or cultural perspective. None can deny the presence of perfection found in the exquisite sphere. Whether at the scale of a planet, a floating soap bubble, or in the size of a pearl, the spherical shape has been associated with perfection and cosmological significance in many ancient cultures. The Renaissance provided us the lens of humanism and allowed for a different perspective in which circles and spheres were perceived. During this period of time, buildings often implemented the dome (half sphere), as this shape was able to deliver both structural performance and symbolic meaning. The dome delivered spatial qualities worthy of mediating the conceptions of the universe and therefore, they were seen as a paragon of shapes that it was possible for humankind to achieve.

The question of whether the sphere was used deliberately as a preconceived form or whether it was merely the result of a constructive system can be asked in relation to sixteenth-century Mexican domes. The conditions in which these vaults emerged suggest that Spanish builders were aiming for efficiency of performance rather than symbolism. Nevertheless, the colonial expansions within Mexico brought radical changes in modes of thought and many of those changes were inspired by the idealized ideas of the European Renaissance. Many of the buildings constructed in Mexico were designed to convey meaning, either spiritual or in regards to the conquest. Architects who traveled to Mexico during the second half of the sixteenth century possessed the necessary academic background to give significance to those hundreds of monuments emerging across the continent. Did the sphere, as an architectural shape, play a role beyond being an efficient shape for construction? Was the sphere used as a design element in order to deliver the 
avant-garde architecture to the new emerging society? Were Renaissance design precepts expressed merely through decorative elements, or did they inform the design composition through geometric and spatial manipulations?

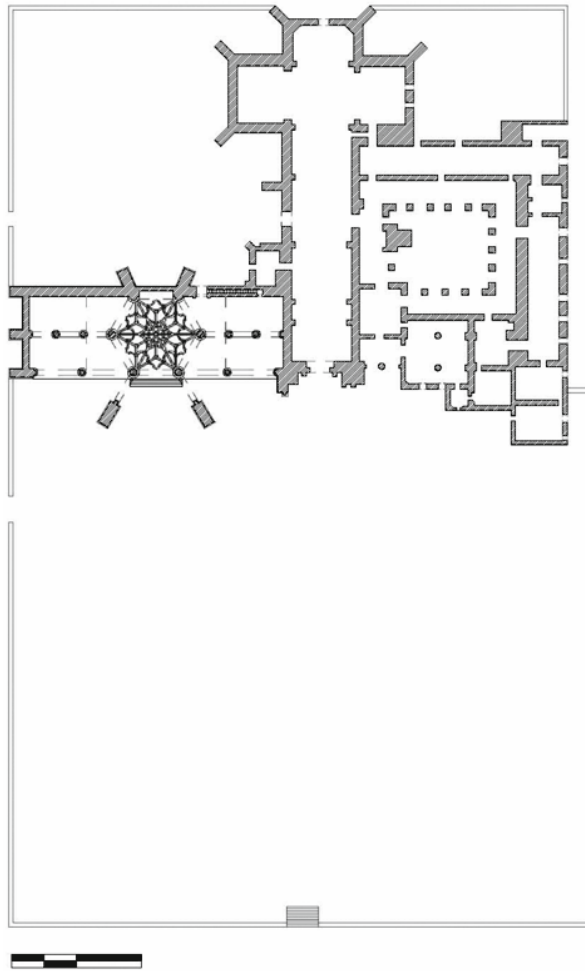

Fig. 1. Plan of the monastery of San Pedro and San Pablo Teposcolula, the open chapel occupies the left wing of the building
The Open Chapel of San Pedro y San Pablo Teposcolula, built between 1550 and 1580, is one of the most refined colonial buildings found in the Mexican territory (fig. 1). This chapel stands out amongst the hundreds of churches, chapels, and other religious buildings that materialized in Mexico in the sixteenth century. The Open Chapel features a unique layout with monumental dimensions creating a spatial and structural configuration that goes beyond the regular forms of the architecture practiced at the time. The chapel emerges in a context of intense building construction activity in the country where thousands of souls were converting to Christianity; this vigorous period provided fertile ground to the creation for this extraordinary building. The spherical ribbed vault with a hexagonal base covering the altar has no precedent in the history of Hispanic architecture. The sphere was used as a means to provide an avant-garde piece of architecture for the emerging town of Teposcolula and its inhabitants.

\section{Open chapels in colonial Mexico, the Chapel at Teposcolula as one of a kind}

The fusion of cultures that took place during the sixteenth century in the Americas led to substantial social changes. The transformation of pre-Hispanic institutions involved the simultaneous transformation of Europe: the configuration of the latter is indigenized and the detailed repertory of the former is Europeanized. ${ }^{1}$ Architecture built during this period of time reflected this melding of cultures, creating unique archetypes such as the open chapel, a building type designed to accommodate both the indigenous and Christian rituals. One particular aspect of the indigenized Christian rituals is that they took place using an exterior space, such as a square or plaza. The open chapel typology made it possible to merge both traditions by uniting a covered space that worked as an altar with a wide-open space facing it. This new type of building emerged naturally as a consequence of the historic circumstances and its novelty made it subject to architectural experimentations. $^{2}$

Mexican art historian Manuel Toussaint (1890-1955) was the first to introduce the term open chapel. He described this type of building as the only possible analogy between the Christian church and indigenous teocalli (sanctuary). ${ }^{3}$ The atrium, the open 
space found within many of the monastic complexes in Mexico, played the role of the true church, and the chapel was intended only as an altar to be used by priests, the choir and the main congregation. The system of the atrium or the open chapel was generous and tolerant of indigenous concepts of space and resonant with the ancient rhythms of both the Christian and the indigenous ritual. ${ }^{4}$ Several examples of open chapels are found throughout the Mexican territory. This type of building was an effective tool in accomplishing the evangelization endeavors of the mendicant orders that spread throughout the territory.
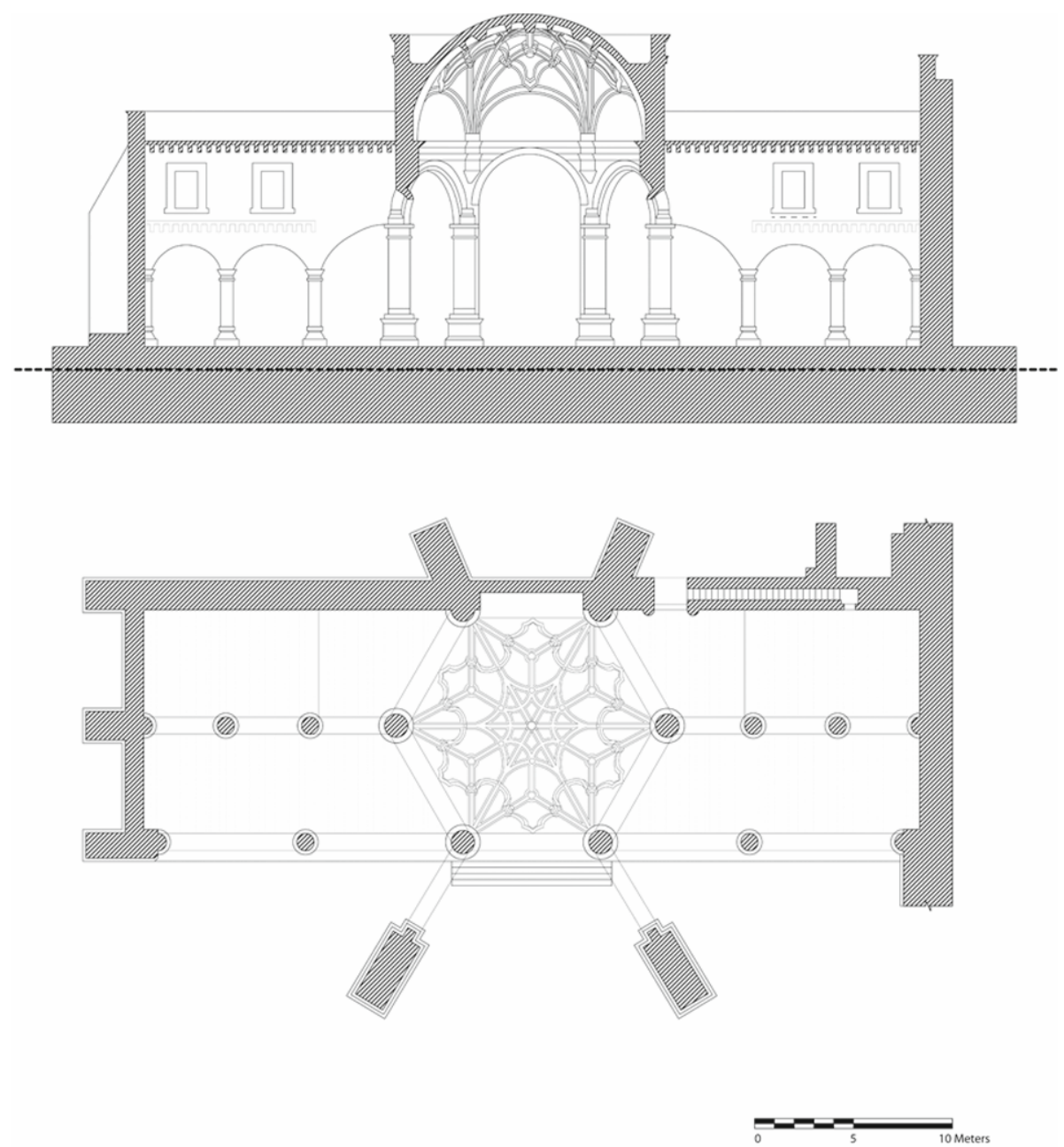

Fig. 2. Plan and section of the Open Chapel at San Pedro y San Pablo Teposcolula

The Open Chapel of San Pedro y San Pablo Teposcolula is a unique building within the family of existing open chapels in Mexico. The building is configured as a single nave that opens its long western facade towards the atrium, where the numerous indigenous people attending the ceremony were positioned (fig. 2). Following a symmetrical scheme, the chapel houses a hexagonal-plan hemispherical ribbed vault that holds an altar. The spaces that flank the dome to the north and south are covered with a roof system of 
wooden beams. A significant feature of this building is that many of its supporting elements are columns, creating a very open floor plan. The back and side walls reinforce the structure while forming a rectangle that serves as the backdrop for activities carried out in the chapel. The long rectangle enclosure formed by the perimeter walls recalls the idea of a traditional church's nave (fig. 3). This is truly a significant piece of Mexican history and has been recognized by art historians such as John McAndrew, who states that no other more elaborate vault was built in the Americas. ${ }^{5}$ For George Kubler, this Open Chapel is unprecedented in the history of architecture, and is Mexico's most original contribution to the worldwide repertoire of specialized forms (fg. 4). ${ }^{6}$ Unlike other open chapels in Mexico, this building is of a monumental scale and, in combination with the ribbed vault, creates an outstanding piece of architecture

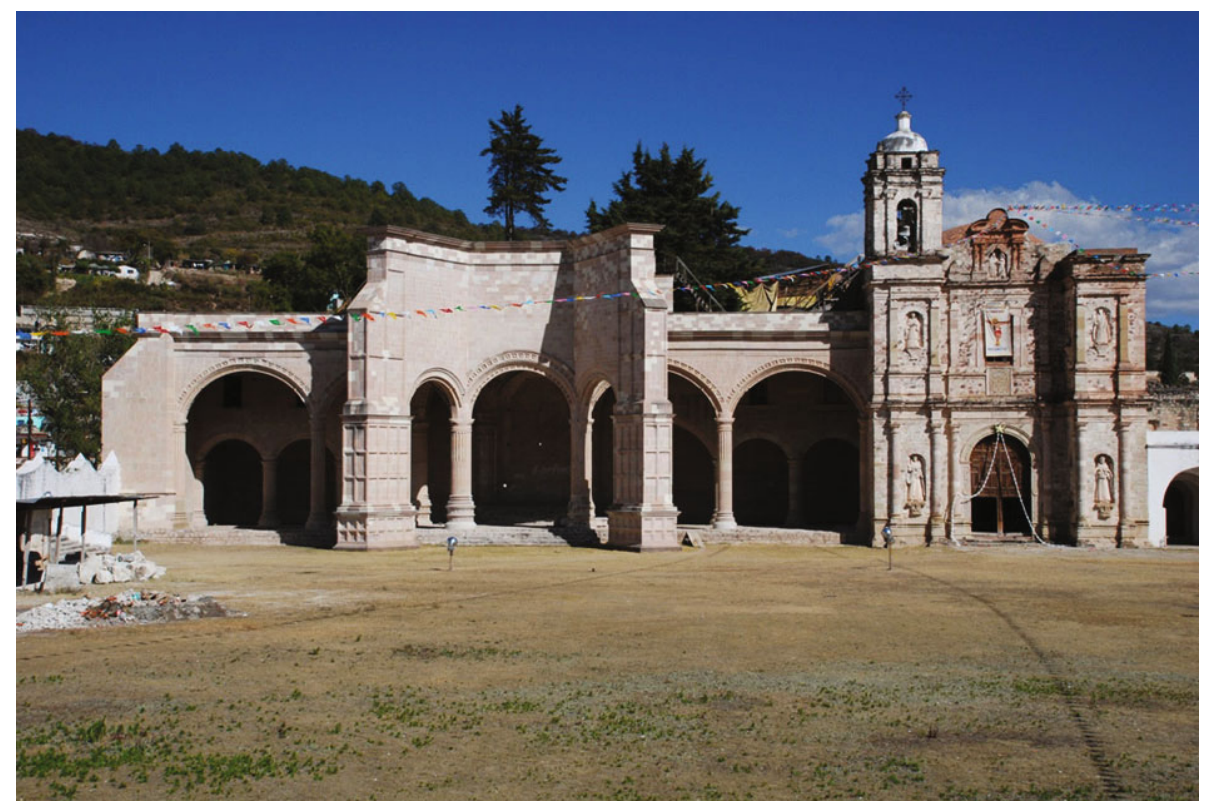

Fig. 3. Frontal view of the Open Chapel at San Pedro y San Pablo Teposcolula

\section{Geometric order, lines, patterns, and harmonic relationships within the Open Chapel of Teposcolula}

Circular and spherical shapes were part of the repertory of forms commonly used during the Renaissance. Even when such conceptual forms were ethereal, architecture was able to solidify them in a geometric armature that lent a physical dimension to them. Robin Evans has established connections between the conceptual understanding of the sphere and its materialization in Renaissance architecture. ${ }^{7}$ Evans makes evident the connections between geometry and architecture, and suggests that the church of Sant'Eligio degli Orefici by Raphael Sanzio is an example of how Italian Renaissance architecture unveils the role of the sphere in architectural design. He proposes that spheres, in fact, might embody symbolic meaning in Renaissance architecture. The architectural scheme of Sant'Eligio contains nine possible points within the space of the church that could be defined as the center. Its dome is a natural response to the centralized character of the building. The design shows how forms embodying sphericity and circularity were held aloft the Renaissance buildings and are not inscribed in the 
plans, positioning centralized churches as symbols of universal order representing the cosmos. This understanding of the cosmos was reflected within numerous dome paintings, which emphasize the spherical layering of the universe and the heavens. For Evans, La Disputa by Raphael, in which imagery is immersed in a spherical space of concentric hierarchy, reveals again this spherical/circular centrality as evidence of the order of the creation and therefore the representation of God. This centralized form of conceiving the cosmos also takes into consideration the vision of Renaissance scientists, who attempted to define an organization and functional model of the heliocentric solar system. For Evans, the architect of Sant'Eligio and the architect of the new universe were dealing with the same problem at the same time.

These formal assertions defined by spatial configurations, lines, and rigorous geometries in architecture are discernable in the realm of mathematics. The sphere and circle can be defined by the relationships established between the form and the position of its center: the center is intimately connected to the sphere, to the extent that their relationship is indissoluble by nature. This relationship is rooted in the realm of geometry, which is often of interest for mathematicians as well as architects, who were equally fascinated by this compelling shape. As expressed by Evans, the architects of the Renaissance had an affinity for the sphere. Centuries later, Stanislaw Ulam, a twentiethcentury Polish mathematician, proposed a problem related to this mythical shape in the Scottish Book, an informal collection of mathematical problems. ${ }^{9}$ Problem 19 reads as follows: "If you place an object of uniform density within water, and it floats in any position, is this object necessarily a sphere?" 10

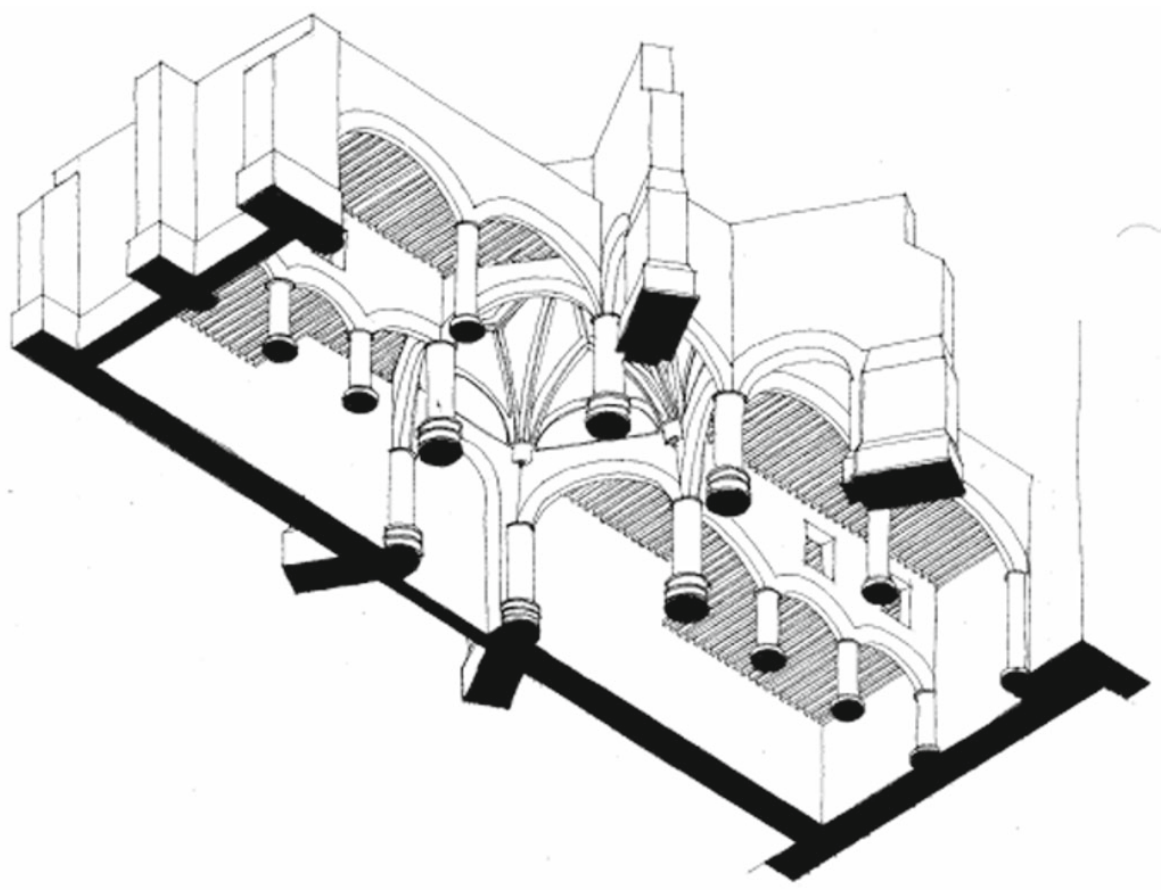

Fig. 4. Axonometric view of the Open Chapel at San Pedro y San Pablo Teposcolula

Depicting the sphere within Euclidian space as the geometric place in which we can observe the properties of the objects in space, Luis Montejano Peimbert, a Mexican 
mathematician, proposed a solution for Ulam's problem. His approach slightly changes the original problem reading as follows: "With a density of zero: A solid may be placed in any position and may rest in equilibrium on a flat horizontal surface. Shall this object be a sphere?" Montejano Peimbert tackled the problem by developing a process through the Convex Bodies Theory. ${ }^{11}$ Sectioning across a sphere and casting shadows to a surface perpendicular to the light rays will produce circumferences that help to determine the spherical shape of an object. Nevertheless, the problem proposed is not completely solved by performing slicing operations. Straight lines, which are useful to define a convex shape, might then be practical in the geometric definition of the sphere. In order to define a convex shape one should first draw the shape on a planar surface, i.e., a piece of paper. In order to verify its convexity one should find two points that are within the figure, these two points should be connected with a line; if the line stays within the figure's boundary then the figure is convex. Another way to identify a convex shape is by drawing a line between two points outside of the figure, the points should force the line to cross through the shape. This line will always intersect two points of the boundary and if it touches only one point then the line is tangent to the shape. The convexity is proven by these operations, but it is still not necessarily a sphere. The spherical figure contains volume and it is therefore necessary to translate the verification process into one that takes three dimensions into consideration. This translation is done based on the parameters of a circumference. All lines tangent to the perimeter of a circumference have a perpendicular that touches the boundary (perimeter) and goes through the center. ${ }^{12}$ This center in space translates as the mass center, which is a point within the solid that behaves as if all the mass would converge in it. This is true for all solids and this point is usually in the very center of the solid. The relationship existing between the supporting (tangent) line and the mass center defines equilibrium. Just as with convex shapes, if a tangent plane (in this case parallel to the horizon) is placed between the mass center and the solid itself then the solid can be in equilibrium. And, in order to place the zerodensity solid in any position and in equilibrium this solid must be a sphere.

As in the process of solving the sphere proposed by Montejano Peimbert, architects and mathematicians also use lines as instruments to solve geometric inquiries. Thus, for architects, lines also serve to assess the sphericity of a building. Elements within buildings are commonly represented by means of a well-defined code or convention, and are usually represented with lines. These lines are both objects contained within a volume, and simultaneously the material of the spatial configurations designed by architects. The Euclidean space in which lines are depicted describes a spatial introspection into the properties of buildings. The ability to translate lines into spatial expressions was intrinsic to the everyday practice of Renaissance architects. The widely studied manuscripts of Alonso de Vandelvira (1580) and Hernan Ruiz (1560) describe the procedures performed to define the geometry of vaults and their arches (fig. 5). ${ }^{13}$ As explained in these documents, the methodology applied to describe the underlying geometry of a ribbed vault began with simple line drawings. The first step was to set out a series of lines that would depict the vault on the horizontal projection, determining its overall proportions and the layout of the ribs. The drawings of the manuscripts also contain the delineation of each arch within the vault on a vertical projection. Each segment of circumference was drawn overlapping the others on the same plane. Although the Vandelvira and Ruiz manuscripts depict hypothetical vaults, the purpose of the drawing was to provide the information useful for controlling the shape and size of the vault. ${ }^{14}$ 


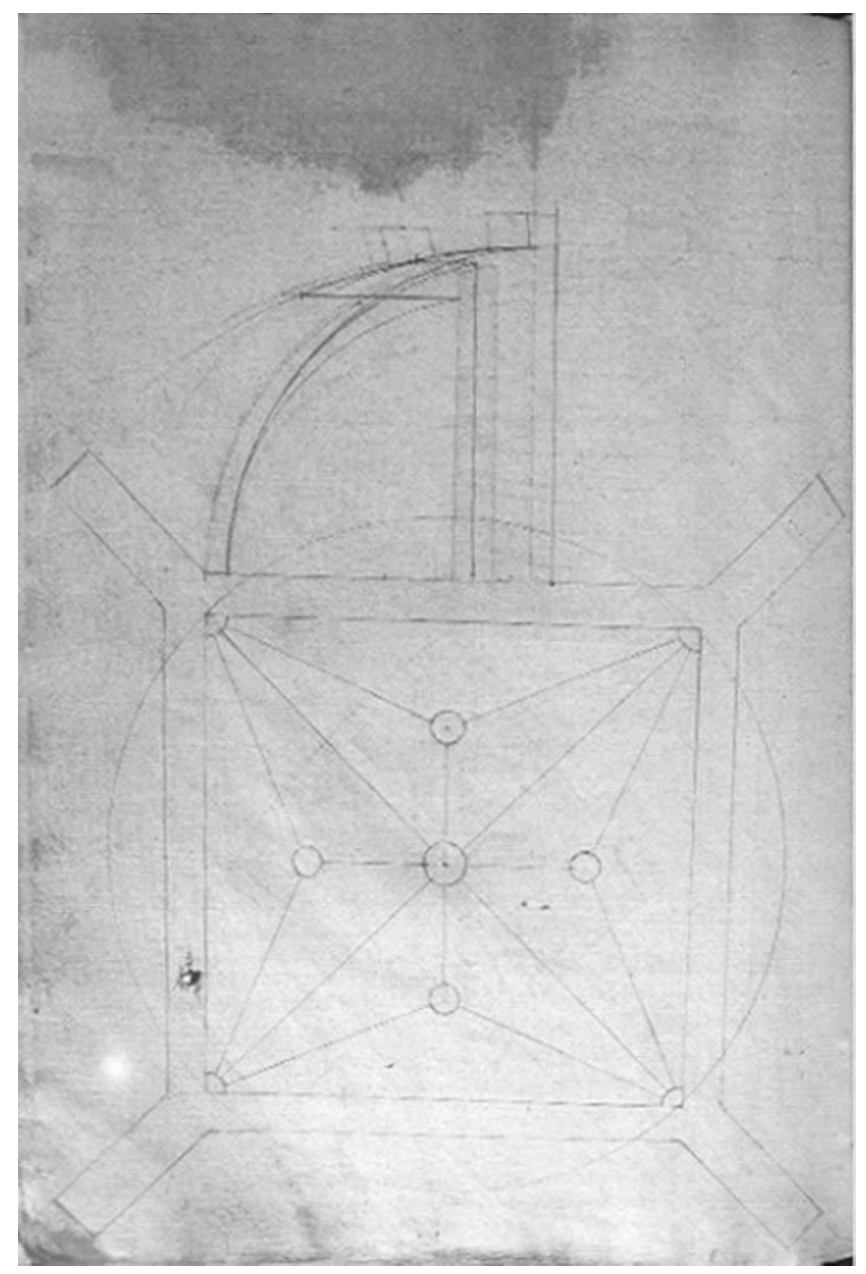

Fig. 5. Drawing by Hernan Ruiz (courtesy of Enrique Rabasa)

The exercise of line drawing was not restricted to the vault alone. Line drawings were used to define the configuration of the entire building. In fact, the tensions existing between these lines and the points holding them provide tools to recreate the design processes. Properties embedded within a building may be observed through twodimensional operations that find their final spatial configurations in the built form. The architectural scheme of Teposcolula's Open Chapel emerges from a layout based on a logic rooted on three hexagons grouped together (fig. 6). This group of hexagons give proportion to the nave in plan and elevation/section simultaneously. The hexagons, as a sequence of lines, provide the platform to define the location and the dimension of other elements of the building establishing a relationship that can be quantified. The composition of the entire building is linked to the hexagon and its geometric relations. Within the polygon located at the very center, one finds the only vault of the building, which creates an organization system based on two different categories. The north and south wings, lower in ceiling height, emphasize the symmetrical composition of the building. The central scheme of the Open Chapel in Teposcolula recalls Raphael's 
church in the repeated elements that correspond to the north and south portions of the building, whose structural, formal, and ornamental characteristics are identical. Both are divided and articulated in turn by the singular centralized space that balances the composition. The sacred space at the center is supported both in volume and form. The two secondary spaces, more measured and lighter, act as a counterweight in the balance whose arms are in perfect equilibrium.
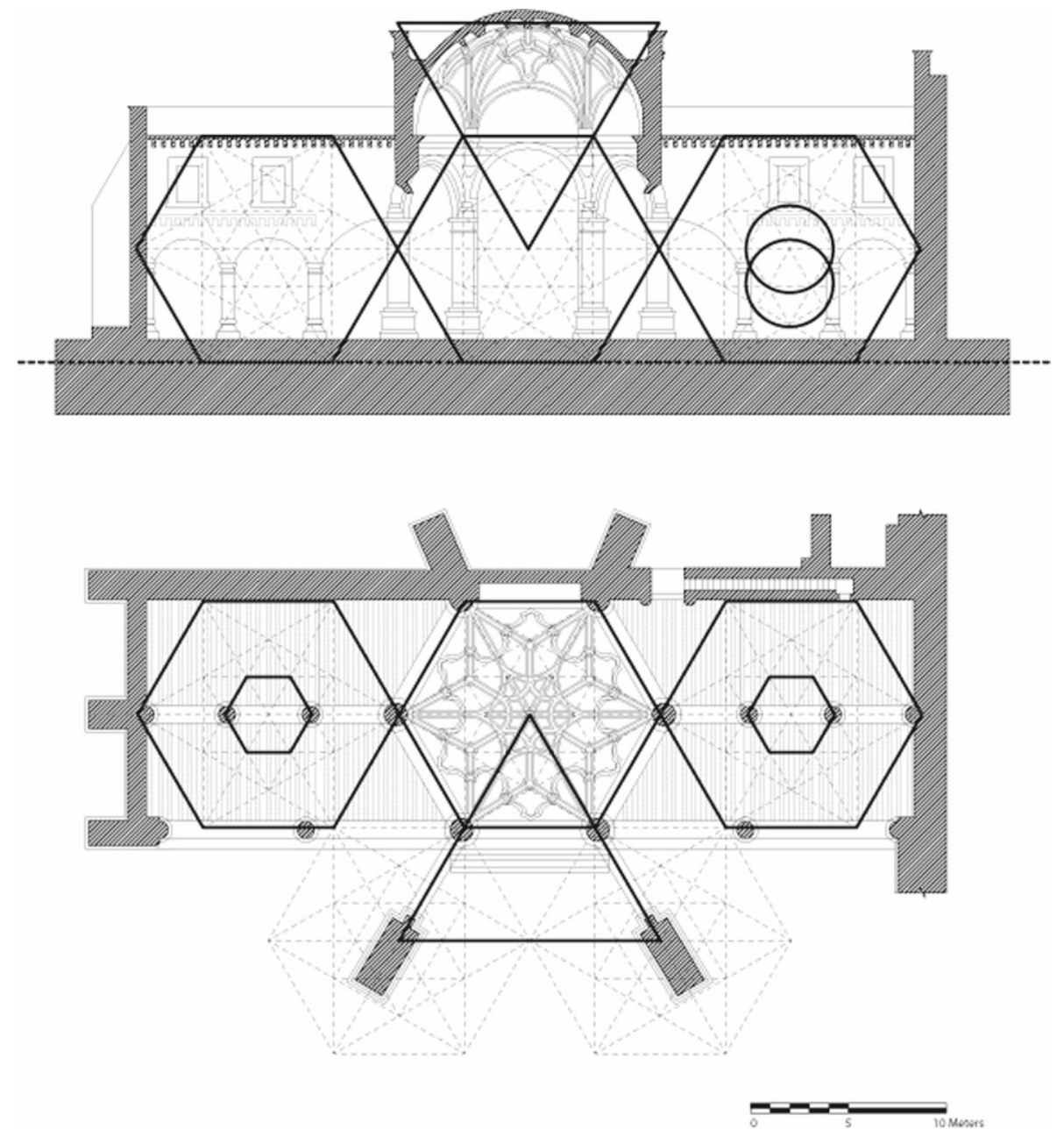

Fig. 6. Diagram showing relationships of plan to section

The Renaissance centralized space of Teposcolula's Open Chapel is based on the correlation between the sphere and the hexagon in plan. Learning first from the hexagon, one understands this almost pure spatial mass density exacerbated by the ornament, arches, and columns that accentuate its relevance. The hexagon is a covered space with sacred character. This central volume, the tallest space of the building, is the predominant space. Spatially it is the richest, containing the symbolic content and supporting the ribbed vault (figs. 7 and 8 ). 


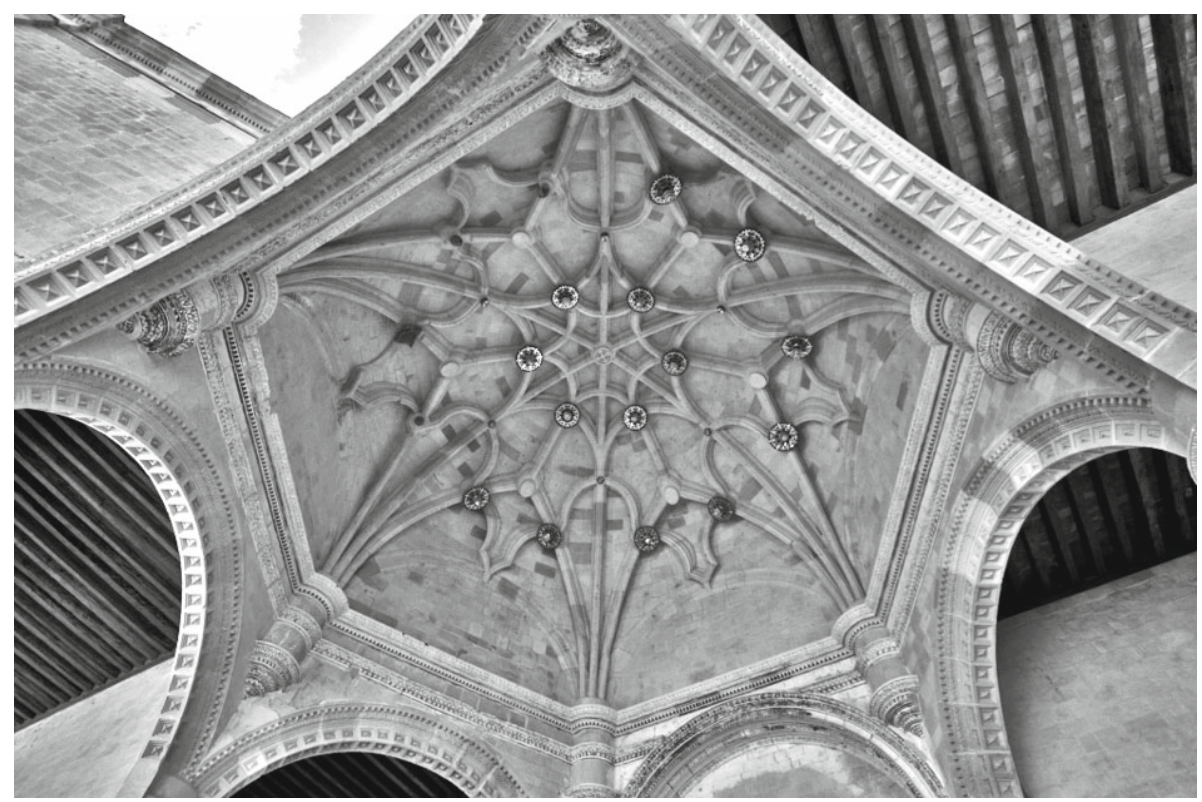

Fig. 7. The vault of the Open Chapel

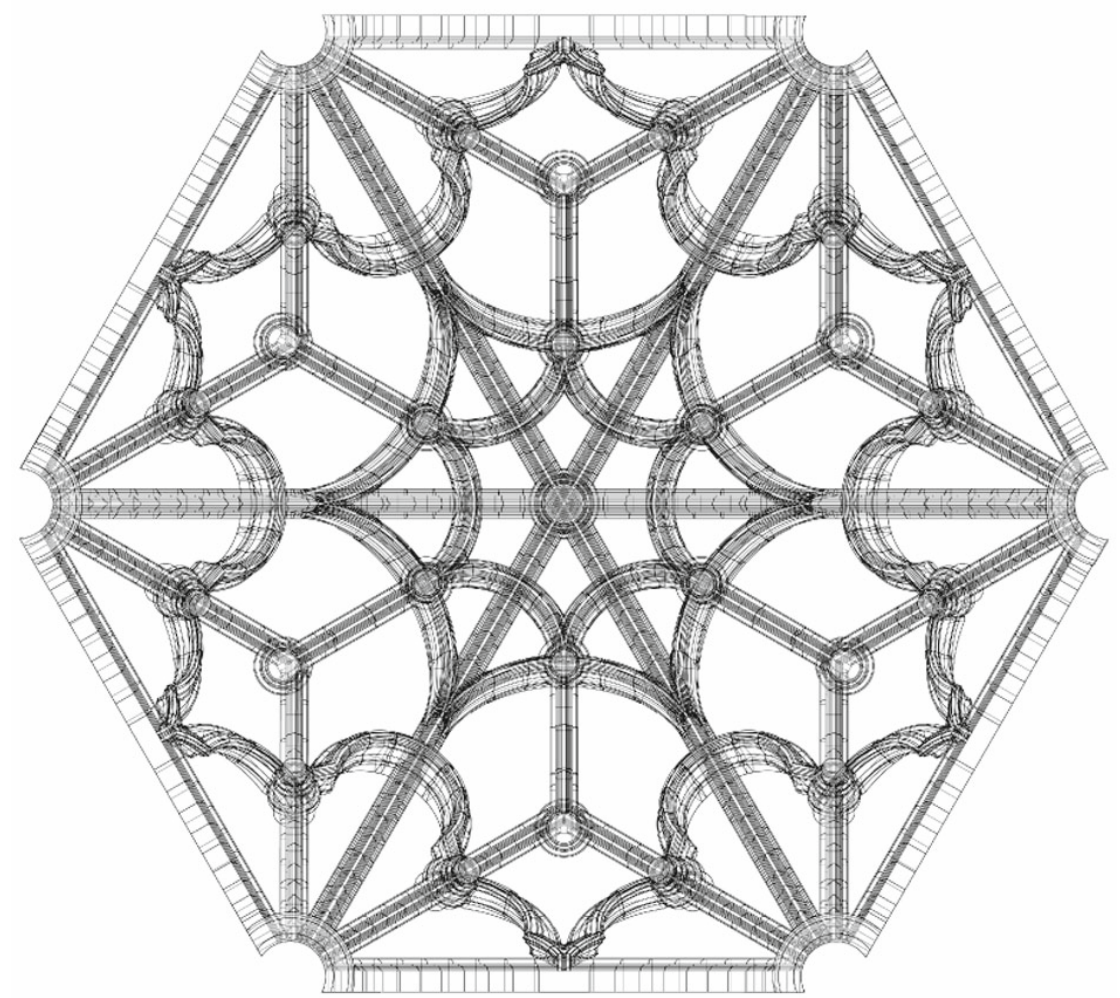

Fig. 8. Reflected ceiling plan of the vault 


\section{Lines defining the sphere, underlying geometry of the ribbed vault}

As Evans would remark, the resolution into circular and spherical forms indicates that centralized churches shared some formal properties with the universe they represented. Following the procedure suggested by the Vandelvira and Ruiz manuscripts, the sequence of lines defining the vault of this open chapel has a twofold result: they serve as a guideline to the construction and emphasize the connection between the inhabitant and the universe. This relationship emerges from linear gestures that accommodate intrinsic relationships creating intricate patterns and polygons. The hexagonal shape is the most important feature of the vault. The underlying geometry is based on this hexagon and the two equilateral triangles that register within, forming the so-called Star of David. By drawing stars within stars, in a progressive sequence that could continue an endless number of times, one can define the position of each individual rib and boss, and even the diameter of the keystone (fig. 9). ${ }^{15}$ The lines drawn within the hexagonal shape are the underlying principle to draw the vault on a horizontal projection. ${ }^{16}$
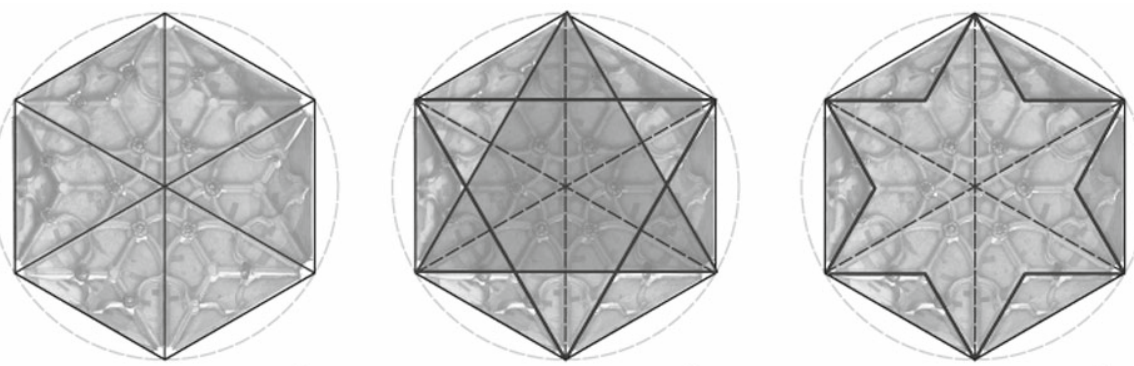

1

2
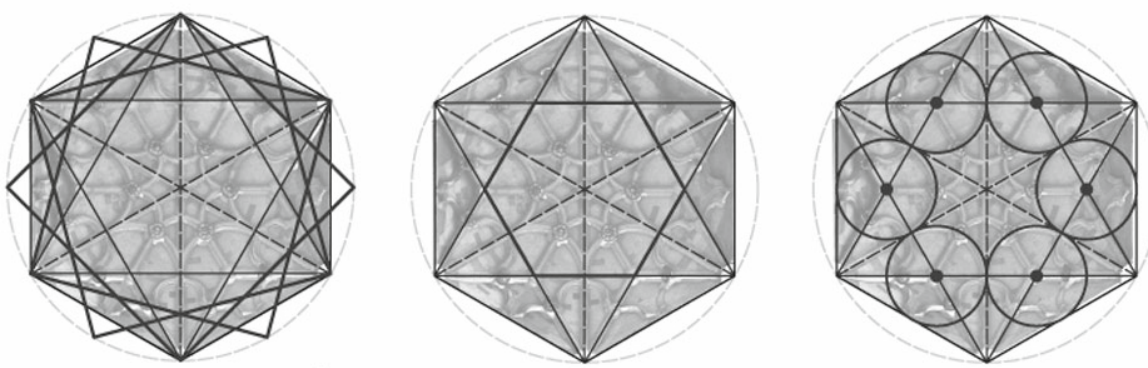

4

5

6
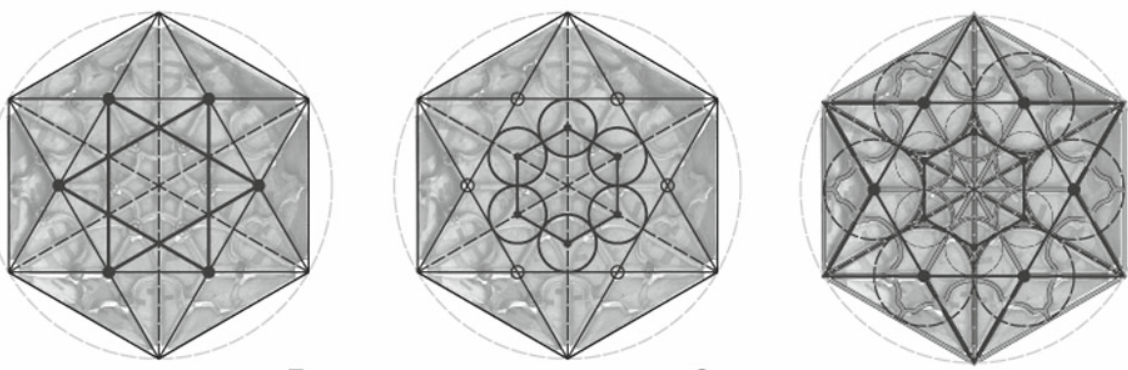

8

Fig. 9. Underlying geometry drawings 
Lines and circles interact in the underlying geometry of the vault in a way that is similar to those serving Montejano Peimbert to define convex shapes (fig. 10). The horizontal lines, hexagons, and the circle reflecting the vault (with radius $A$ on fig. 11) are registered on a projection parallel to the horizon. However, to avoid confusion, it is necessary to clarify that this radius $A$ is not the actual radius of the diagonal arches at the vault's intrados. This is due to a simple reason: stone ribs cannot converge into a single point. Therefore, the vault in the intrados uses a smaller radius and this hexagon is useful only to develop the underlying geometry

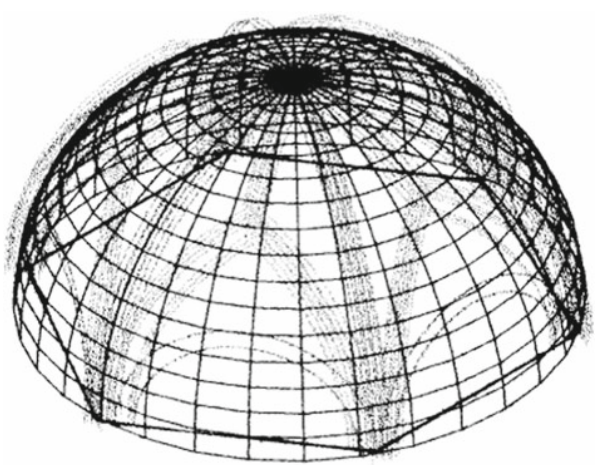

Fig. 10. Spherical surface of the vault of the vault.

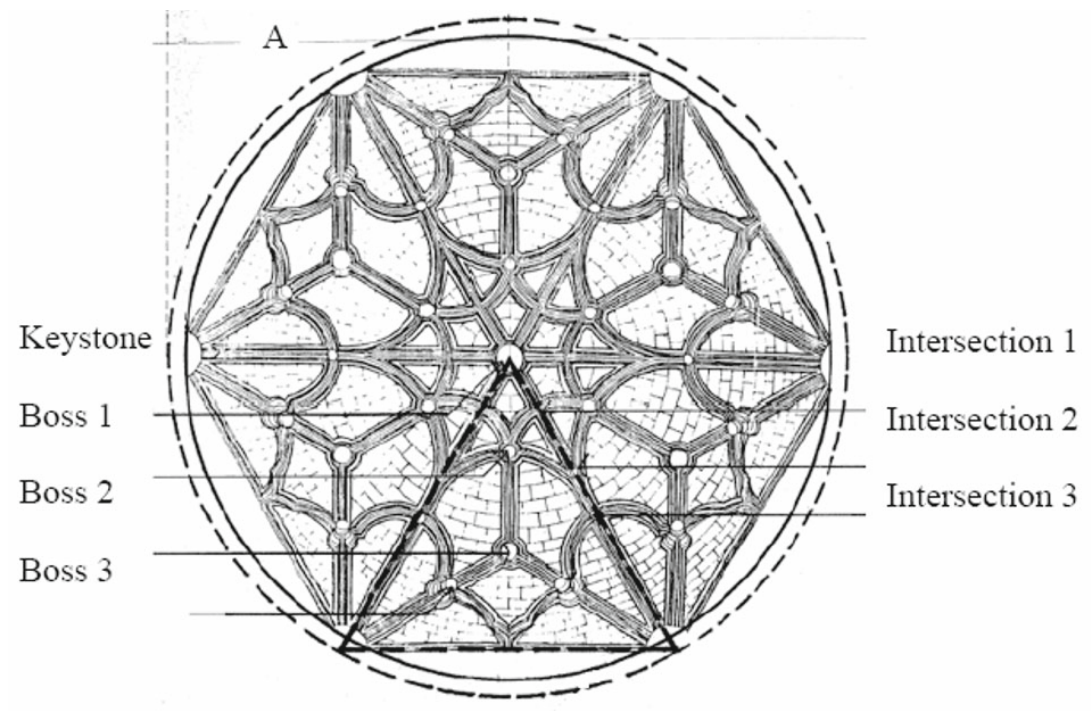

Fig. 11. Diagram showing the parts of the vault, one of the six identical equilateral triangles and the intersections

Once the hexagon is drawn on the horizontal projection, one can distinguish that six identical equilateral triangles are formed, all sharing a corner at the very center. The points defining each triangle are: the center of the circle, and two vertices of the hexagon. These triangles function as a module that is repeated to draw the vault; in other words, it repeats six times within the vault. If the lines define the path of the ribs of the vault, then the points where lines cross each other define the location of bosses and intersection pieces. These pieces are relevant because they articulate two or more ribs, making their stereotomy crucial to the vaults's structural performance. The vault presents two types of solutions for the articulation between ribs: bosses and simple intersections. Fig. 9 shows the sequence of drawings necessary for the vault's underlying geometry description. 


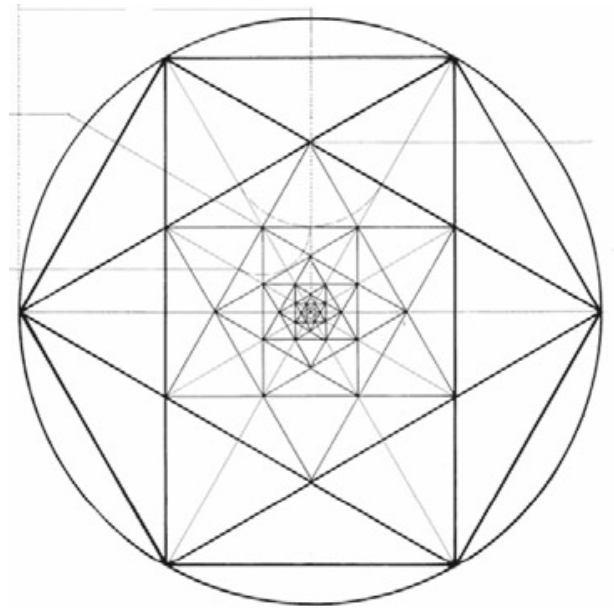

Fig. 12. Star of David within the hexagonal shape

Let us look back to the Star of David drawn within the hexagon. These two equilateral triangles can be drawn within a regular hexagon inscribed in a given circle (the radius of the circle in this case is equal to $A$ ). The intersection points of the sides of these triangles form a smaller hexagon proportional to the first and rotated. As seen in figs. 9 and 12, each smaller hexagon is proportional to its immediate past hexagon and it is clearly possible to repeat this operation progressively until the hexagon becomes a point. This sequence of triangles/hexagons is substantial to the vault's configuration. Tiercerons are drawn from the first internal equilateral triangles (which is also the bisectrix between the diagonal and the former arches). The intersection of the sides of these first triangles provides the position of boss no. 2 and drawing the smaller hexagon/triangles defines position of boss no. 1. These points where the bosses are located are also centers for the semicircles of warped ribs no. 2 and no. 3 .

Looking at the smaller hexagons formed by the sequence of lines and triangles mentioned before, one can find a geometric relationship that may also be repeated until the line becomes a point in the drawing. This relationship relates to the length of the sides of the smaller hexagons (which are inscribed and rotated) in fig. 13. Looking at this figure, one can identify a right triangle whose cathetus is a side of the hexagon base $A$ and whose hypotenuse $H$ forms an angle of $30^{\circ}$ with $A$. The length of the immediately smaller hexagon is half of the length of $H$. Furthermore, $H / 2$ is equal to the dimension of the short cathetus, which dimension has been expressed as $A / \sqrt{3}$. Further development of this drawing reveals that, taking as center the mid-point of $H$ and taking as radius a vertex of the right triangle, one finds a circle in which the triangle is inscribed. This circle will also go through the center of the dome. By using trigonometry we know that the triangle's long cathetus $(A)$ and the angle between this catetus and the hypotenuse is $30^{\circ}$, the dimension of the immediate rotated hexagon $(h)$ can be defined as follows: $H=A / \cos 30^{\circ}$, so that $h=\frac{1}{2}\left(A / \cos 30^{\circ}\right)$. The short cathetus of this triangle is equal to $A / \sqrt{3}$, which leads us to match: $\frac{1}{2}\left(A / \cos 30^{\circ}\right)=A / \sqrt{3}=h$. 


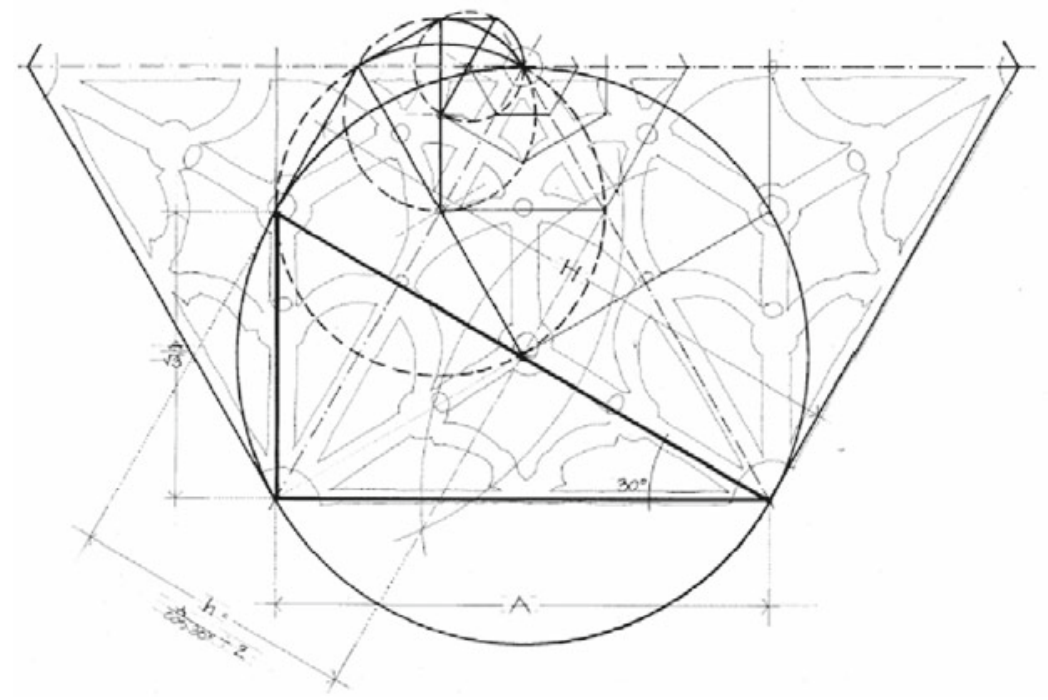

Fig. 13

In fig. 14 we observe that the center of boss no. 2, (the midpoint of the hypotenuse of the triangle mentioned before), is the center for the arc defining the warped rib no. 2 . The circle is inscribed within the equilateral triangle formed by two diagonal arches and the former arches. The relationship between this triangle and the circle's radius is obtained as following: if half of $A$ is $a$, the radius of the warped rib no. 2 responds geometrically: $a / \sqrt{3}$.

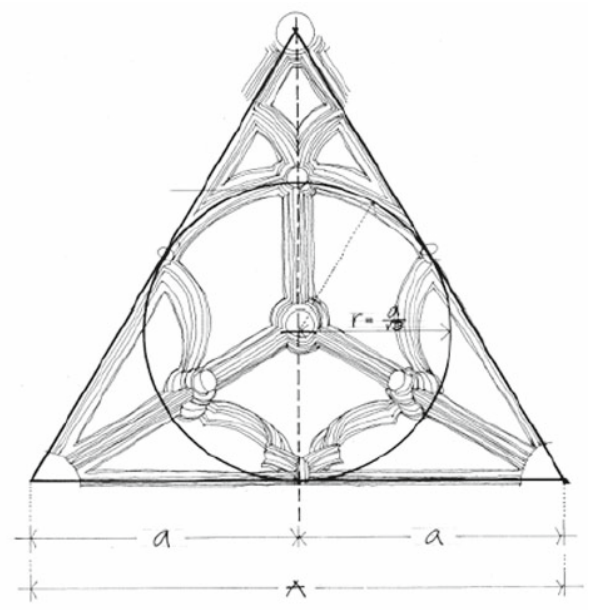

Fig. 14

Now, going back to the initial hexagon and the two equilateral triangles forming the Star of David, we draw three squares inscribed in the same circle. 
In fig. 15 we observe that those three squares are placed so that two corners of each square coincide with two vertices of the hexagon. The lines connecting the squares' vertices create a dodecagon. This regular twelve-sided figure allows for a combination between a system based on number 3 (as in a triangle) and a system based on number 4 (as in a square). This insertion of the dodecagon is necessary to draw the vault because the bosses no. 3, which are embedded in the tiercerons, are located where the sides of the squares intersect the sides of the equilateral triangles.

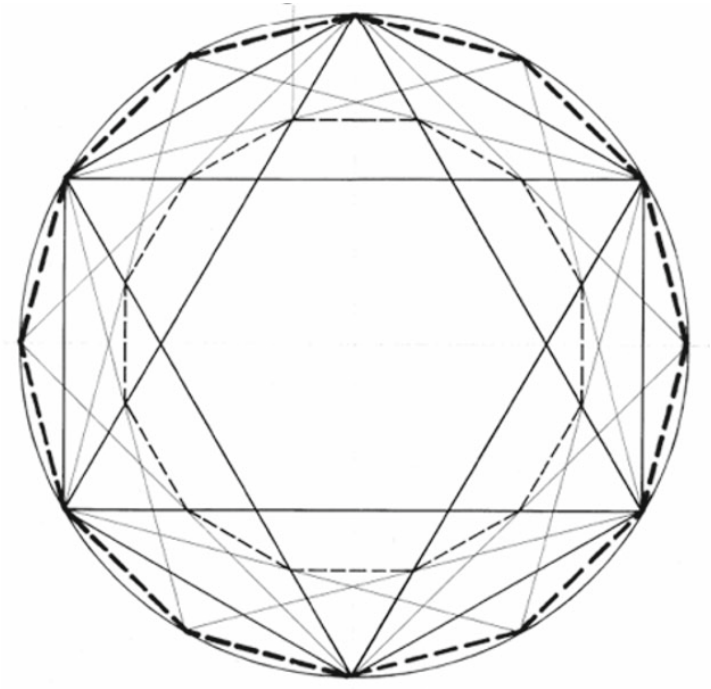

Fig. 15. Squares inscribed within a hexagon

Now, by connecting the points with a line where these bosses are located, we obtain a polygon with twelve sides, proportional and rotated with respect to the initial dodecagon, similarly to the hexagon drawings seen before. The bosses no. 3 are equidistant from each other forming a regular polygon of twelve sides. We will see in the following principle that, in addition to this relationship, bosses no. 3 have a proportional position in relation to bosses no. 2 .
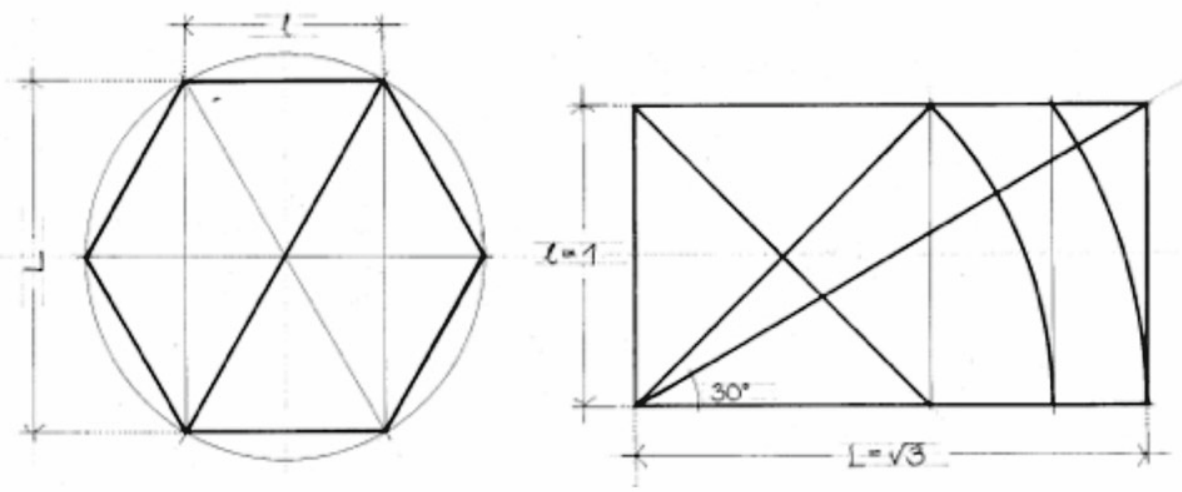

Fig. 16 


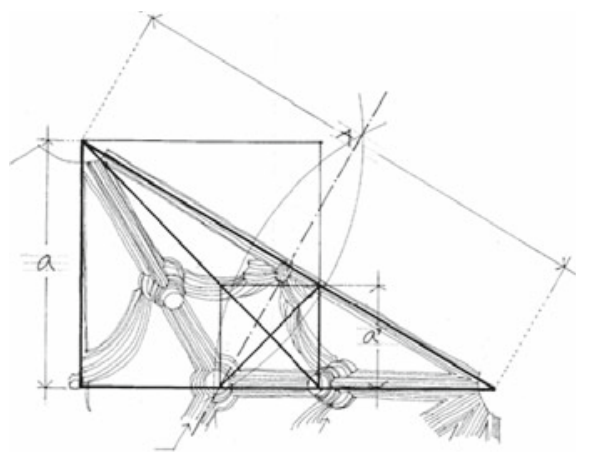

Fig. 17

In fig. 16 we observe a right triangle formed by internal angles at $30^{\circ}, 60^{\circ}$, and $90^{\circ}$. This triangle is used to draw a rectangle of $\sqrt{ } 3$ proportion and is the base of subsequent figures such as a hexagon. By assigning values to the sides of this triangle when $l$ equals 1 , $L$ is $\sqrt{3}$. Finally, we observe this triangle from a different perspective within the vault. As represented in fig. 17, we find that the hypotenuse of the triangle is one side of the hexagon's base. This hypotenuse is now equal to $A$ and its short cathetus is equal to $a$. Drawing a line perpendicular to the midpoint of the hypotenuse, we find that the point where it crosses the long cathetus, is the point defining the location of boss no. 2. From this last point, we draw a $45^{\circ}$ line to intersect the hypotenuse and construct a square inside the triangle whose side is $a^{\prime}$. One of the corners of this square defines the location of boss no. 3. This sequence of lines can be repeated many times inside and outside of the triangle.

The square with $a^{\prime}$ as side length is the geometric figure relating to the location of bosses no. 2 and no. 3 . In fig. 18 we can observe that $a^{\prime}$ creates relationships within a portion of the vault, defining points of relevance for a rectangle whose sides are $A / \sqrt{ } 3$ and $A$. First we find that $A / \sqrt{3}=a^{\prime}+a^{\prime} \sqrt{3}$. Looking back to fig. 17 we determined $a$ as $A / 2$. Now, in this same figure, we see that $2 a^{\prime}+2 a / \sqrt{ } 3=A$ and therefore $2 a / \sqrt{ } 3=A / \sqrt{ } 3$. As conveyed in this drawing, this can be determined by a sequence of rectangles and squares derived from the right triangle seen earlier in fig. 13.

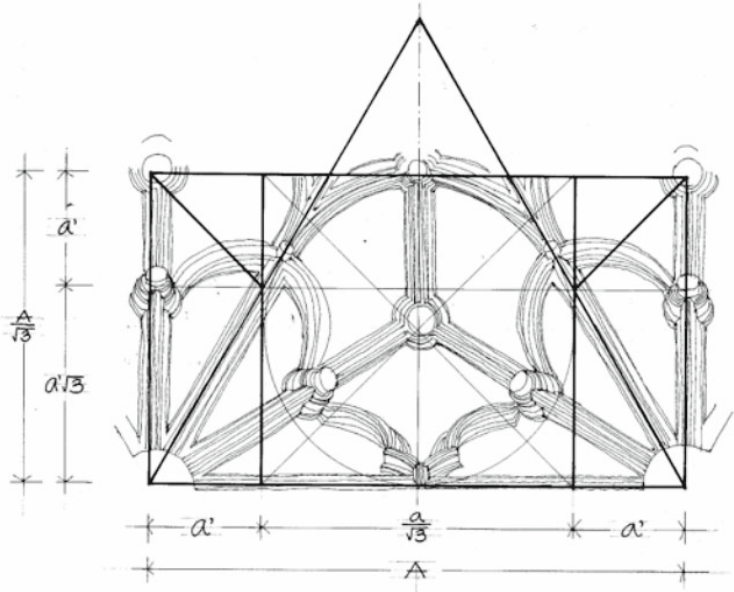

Fig. 18 


\section{Spatial configurations beyond the two-dimensional patterns}

Through these sequences of figures we have been able to identify lines and shapes defining different geometric relationships within the vault. These series of abstractions remind us of the conceptual frame of architectural design, which occurs regardless of material considerations. The architect of the Renaissance worked with abstract means of design using a wide repertory of geometric figures. ${ }^{17}$ These geometric forms are the media to convey shape from conceptual representation to material construction. This construction appears in a spherical shape that, as Evans proposed, shared formal properties with the universe they represented. ${ }^{18}$ Because this particular vault was constructed in Mexico, it is pertinent to formulate the question: Was the intention of the designer to deliver meaning through the construction of the vault? If so, who was this directed towards? The indigenous people who were being evangelized? The Spaniards who were familiar with the Jewish-Christian tradition? We should remember that the symbolic connotations conveyed by the vault took place during an agitated time in history when profound changes were taking place. There is no doubt that this transformation of the social environment provided the ideal opportunity for architectural experimentation. Geometry, on the other hand, provided the palette of forms to generate architectural composition. This palette was loaded with Gothic praxis, such as that found in the notebook of Villard de Honnecourt, whose drawings clearly express that the mindset of the medieval builder had to be geometric. This form of design practice simultaneously infused meaning into constructive and structural solutions. ${ }^{19}$ For the designer of the Open Chapel this medieval background influenced a series of geometric attitudes and constructions informed by resolutions loaded of symbolic meaning.

On paper, combinations of prismatic, cylindrical, and spherical volumes govern the basic shape of the building. Their scale, ratio, and frequency is regulated through numerical rules. Nevertheless, reading the vault with its spatial characteristics necessitates a second layer of abstractions. Reviewing Marsilio Ficino's demonstrations on the immaterial essence of aesthetics, architecture may be read by abstracting the matter in our thoughts, leaving order suspended. ${ }^{20}$ This notion reinforces the conceptual proposition of these spherical surfaces floating above the corporeal substance of the centralized churches. Ficino's invitation to abstraction becomes the connecting vehicle for translating lines revealed through this study into lines that travel through the spherical space of the Open Chapel's vault. These geometric abstractions will exceed the geometries based on circles, cylinders and spheres, encouraging us to perceive the vault as Carl Sagan suggests that we read the most common constellations of the celestial sphere, that is, by imagining their exact position within the space of the universe, rather than as they are drawn on a twodimensional star chart. What this text suggests is to hold the imaginary sequence of lines seen in the exposed drawings and to place them within the surface of the sphere, decomposing and/or recomposing trajectories from point to point. This visualization, in fact, brings to mind the solution to Ulam's problem by Montejano Peimbert, which is defined by a mathematical interpretation. This scientific approach might serve to prove an idea that is not scientific in nature by merging this proposition within the context of architecture and reflecting spatial points and lines within the underlying principles of the vault of Teposcolula.

The imaginary set of points that represent the centrality of Sant'Eligio are a question of symbolism. These points of centrality in Teposcolula are divergent and inscribed within the sphere following the theory of convex shapes. As the geometric solution to the sphere in equilibrium does, the points located in the chapel's vault define the sphericity 
by drawing lines within the vault itself. The lines on the horizontal projection are cast as the shadows of complex shapes in order to confirm the existence of the sphere. This mental abstraction exercise proposed by Ficino within the context of the Ulam's problem transforms the understanding of the sphericity, which is held aloft and is not inscribed in the plans. The means of representation suggested through orthographic projections are rendered into spatial qualities relying on the ability to translate lines into volume.

The Open Chapel of Teposcolula, similar to Sant'Eligio degli Orefici, presents a scheme where centrality is emphasized by the use of the sphere. This Open Chapel is testimony to how this building technology was imported to the Americas. Furthermore, it reveals how the integration of European building technology with Mexican indigenous artisanship gave origin to outstanding architectural models inspired by the spirit of the Renaissance utopia. The construction processes were ruled by the structural constraints and the results were indeed state-of-the-art for their time. Setting aside symbolism and, in some extent, being of pure mathematical speculation, the design of the Open Chapel of Teposcolula was anchored in the significance of local interpretation of constructive principles by stating the symbiotic process of indigenous masonry techniques and European applied geometry. In this context, we should remember that the vicissitudes that took place during the sixteenth century in Mexico created a challenging environment for the constructions of such a sophisticated nature. ${ }^{21}$

\section{Notes}

1. George Kubler's book covers a good amount of the buildings in Mexico during the sixteenth century. His conclusions remark the merging of the indigenous and the Spanish cultures [1985: 537].

2. My text published in the proceedings of the 7th Spanish Congress on Construction History provide a more detailed information on the novelty of the buildings constructed in the Mixteca during the second half of the sixteenth century [Ibarra-Sevilla 2011].

3. Manuel Toussaint is a pioneer of the studies on sixteenth-century architecture in Mexico [1983: 13].

4. Kubler dedicates a portion of his text to the study of the open chapels [1985: 538].

5. John McAndrew dedicates an entire volume to the study of the open chapels in Mexico. He categorizes the different buildings and expresses his admiration for the chapel in Teposcolula [1965: 545-555].

6. Kubler emphasizes in his text the unique character of Teposcolula's chapel [1985: 537].

7. The work of Robin Evans is characterized by his profound understanding of geometry and the ability to associate, almost naturally, the conceptual framework of his inquiries with art and buildings; see [Evans 1995: 3-47].

8. Evans navigates through the cosmology of the Catholic faith and Ptolemaic astronomy, ventures on the fascinating layering of Dante's Divine Comedy, identifying Dante as the great synthesizer of figures: figures as persons, figures as numbers, and figures as shapes; see [Evans 1995: 19].

9. The book takes its name from a coffee shop located in Lvov, Poland, where mathematicians used to get together and discuss problems of mathematics. In 1935 Stefan Banach (18921945), a professor of Lvov University, proposed recording the multiple discussions and provided a notebook where they were able to write down those problems discussed while sipping a cup of coffee. A waiter of the shop kept the book, he knew the ritual and he would bring it to the table when Banach or Stanislaw Mazur (1905-1981) showed up. Stanislaw Ulam (1909-1984), a young mathematician in the group, proposed problem number 19 related to the spheres, which will be mentioned later in this text. According to Ulam, some of the problems were solved immediately after they were proposed; some other problems are still under discussion. Ulam describes the environment in which this book was written as informal, 
with some tinges of bohemian spirit, and remarks that the discussions that took place in that coffee shop were very enriching, and distinguished by their collaborative character.

10. This question has been translated in a different way for this text. The original text formulates the question as follows: Is the solid of uniform density floating in water in any position a sphere?

11. Luis Montejano Peimbert is a professor of mathematics at the National Autonomous University of Mexico. The solution he proposed for this problem appears in his book La Cara Oculta de las Esferas [1989].

12. This last proposition connects to the definition of the circle, its diameter and the relationships with convex figures that are not precisely circular but share some of the characteristics of the circle. The final solution, which begins with a cylinder of uniform density, which will float on water on any position parallel to the generatrices of the cylinder. Montejo-Peimbert gives allusion to Zindler curves, which describe irregular figures in which all the cords cutting the area in half have the same magnitude. Prisms that use these curves as directrices would float in equilibrium when density is half. These directrix curves are not necessarily symmetric and would thus not necessarily form a cylinder [1989: 61-82].

13. For more detail on the analysis and description of Ruiz and Vandelvira manuscripts see the work of two professors at the Polytechnic University of Madrid: Jose Carlos Palacios [2003: 287-321] and Enrique Rabasa [2000: 124-128].

14. It is important to note that Vandelvira and Ruiz use a vault of square plan to demonstrate their drawing methods. According to Javier Gómez Martínez the square was the simplest figure as all its sides are equal and therefore similar solutions will apply to all panels within the vault [1998: 59].

15. The study is supported by rigorous documentation of the vault through laser scanner. The images in fig. 9 are obtained from this survey project. For more information on the results of the scanner laser survey, see [Ibarra-Sevilla 2011].

16. The geometric approach explained here relies on graphic information conveyed through the diagrams joining this text. The lines represent the path of ribs and these lines locate the intersections in the vault where the bosses are located.

17. According to Evans, architects "were able to work, as no other artist had, directly with the most mercurial of all media: geometric figures" [1995: 37].

18. Evans's inquiries are based on the ideas of two art historians, Heinrich Wölfflin and Rudolf Wittkower, related to centralized churches in the Renaissance. Evans contrasts the two positions, one claiming the rational, humanistic and aesthetic aspirations of the centralized buildings, and the other claiming the ulterior reality of this architecture based on its symbolism. This dichotomy is the ground for Evans to develop his argument [1995: 3-18].

19. Carlos Chanfón Olmos, a Mexican professor, made a study of Villard's manuscript. To date, this book is the only complete interpretation of the manuscript available in Spanish language [1994: 27] .

20. Evans reviews with more detail the proposition of Ficino as a means to understand the manipulation of geometric shapes in Renaissance architecture [1994:38].

21. I provide more details about the historic circumstances that took place during the sixteenth century in the region where the Open Chapel was built. The text is found in the proceedings of the 4th International Congress in Construction History [Ibarra-Sevilla 2012: 393-400].

\section{References}

Chanfón Olmos, C. 1994. Wilars de Honecort su Manuscrito. Colección Mexicana de Tratadistas. UNAM México.

Evans, R. 1995. The Projective Cast. Cambridge, MA: MIT Press.

Gomez Martinez, J. 1998. El Gótico Español de la Edad Moderna. Valladolid: Universidad de Valladolid.

IbarRa-SeVIlla, B. 2011. La Cantería Renacentista de la Mixteca. Análisis Estereotómico de Tres Bóvedas Nervadas en Oaxaca, México. Pp 674-685 in Actas del Septimo Congreso Historia de la Construccion, Santiago Huerta, ed. Madrid: Instituto Juan de Herrera. 
2012, Transmission of Building Technology from Europe to the Americas: Underlying Geometry and Stereotomy Studies of Three Ribbed Vaults Constructed in Mexico between 1535 and 1575. Pp. 393-400 in Nuts \& Bolts of Construction History: Culture, Technology and Society, Robert Carvais, et al., eds. Paris: Editions A\&J Picard.

Kubler, G. 1985. Arquitectura Mexicana del siglo XVI. Mexico City: Fondo de Cultura Económico.

MCAndrew, J. 1965. The Open-Air Churches of Sixteenth-Century Mexico. Cambridge, MA: Harvard University Press.

Montejano Peimbert, L. 1989. La Cara Oculta de las Esferas. Mexico City: Fondo de Cultura Económico.

Palacios, J. 2003. Trazas y Cortes de Cantería en el Renacimiento Español. Madrid: MunillaLería.

Rabasa, E. 2000. Forma y Construcción en Piedra, de la Cantería Medieval a la Estereotomía del Siglo XIX. Madrid: AKAL.

Toussaint, M. 1983. Paseos Coloniales. Mexico City: Fondo de Cultura Económico.

\section{About the author}

Benjamin Ibarra-Sevilla is currently a Research Fellow at the Center for World Heritage Studies (CWHS) and Assistant Professor of Architecture at the University of Minnesota. He is an architect graduated from Autonomous National University of Mexico with Merit Award and he holds a Degree in Building Conservation from the Excellence Program of the Carolina Foundation and the University of "Alcala de Henares", Spain. His expertise involves case studies of ancient mason techniques, stereotomy, descriptive geometry and architectural geometry informed by formresistant structures. His work in masonry, geometry and stereotomy has been published numerous times and he has lectured in different academic institutions of Mexico, Uruguay, Argentina, Spain and the U.S. His research focuses on the transmission of building technology from Europe to the Americas making emphasis on the constructive and geometric analysis of sixteenth-century ribbed vaults. 